\title{
Care planning needs of palliative home care clients: Development of the interRAI palliative care assessment clinical assessment protocols (CAPs)
}

\author{
Shannon Freeman ${ }^{1 *}$, John P Hirdes ${ }^{2}$, Paul Stolee ${ }^{2}$, John Garcia ${ }^{2}$, Trevor Frise Smith ${ }^{3}$, Knight Steel ${ }^{4}$ and \\ John N Morris ${ }^{5}$
}

\begin{abstract}
Background: The interRAI Palliative Care (interRAI PC) assessment instrument provides a standardized, comprehensive means to identify person-specific need and supports clinicians to address important factors such as aspects of function, health, and social support. The interRAI Clinical Assessment Protocols (CAPs) inform clinicians of priority issues requiring further investigation where specific intervention may be warranted and equip clinicians with evidence to better inform development of a person-specific plan of care. This is the first study to describe the interRAl PC CAP development process and provide an overview of distributional properties of the eight interRAI PC CAPs among community dwelling adults receiving palliative home care services.
\end{abstract}

Methods: Secondary data analysis used interRAI PC assessments $(N=6,769)$ collected as part of regular clinical practice at baseline $(N=6,769)$ and follow-up $(N=1,000)$. Clients across six regional jurisdictions in Ontario, Canada, assessed to receive palliative homecare services between 2006 and 2011 were included (mean age 70.0 years; \pm 13.4 years). Descriptive analyses focused on the eight interRAI PC CAPs: Fatigue, Sleep Disturbance, Nutrition, Pressure Ulcers, Pain, Dyspnea, Mood Disturbance and Delirium.

Results: The majority of clients triggered at least one CAP while two thirds triggered two or more. Triggering rates ranged from 74\% for the Fatigue CAP to less than 15\% for the Delirium and Pressure Ulcers CAPs. The hierarchical CAP triggering structure suggested Fatigue and Dyspnea CAPs were persistent issues prevalent among the majority of clients while Delirium and Pressure Ulcers CAPs rarely trigger in isolation and most often trigger later in the illness trajectory.

Conclusion: When any of the eight interRAI PC CAPs are triggered, clinicians should take notice. CAPs triggered at high rates such as fatigue, dyspnea, and pain warrant increased attention for the majority of clients. Consideration of triggered CAPs provide evidence to inform a collaborative decision making process on whether or not issues raised by the CAPs should be addressed in the plan of care. Integrating evidence from the interRAI PC CAPs into the clinical decision making process support care planning to address client strengths, preferences and needs with greater acuity.

\section{Background}

Palliative care prioritizes the management of severe, unpleasant symptoms, especially pain, to improve quality of life (QOL). Quite often, but not always, this effort is directed to the care of persons with a life limiting illness. Palliative care also strives to improve the sense of wellbeing of the person's informal support network, including family members and other caregivers [1]. At the most

\footnotetext{
* Correspondence: shannon.freeman@unbc.ca

${ }^{1}$ School of Health Sciences, University of Northern British Columbia, 3333

University Way, Prince George, British Columbia V2N 4Z9, Canada

Full list of author information is available at the end of the article
}

basic level, palliative care may be "achieved through prevention and relief of suffering by means of early identification, comprehensive assessment, and treatment of pain and physical, psychosocial, or spiritual problems" [2].

With support from informal care networks, community based palliative services have made dying at home increasingly accessible to persons with a life limiting illness. Palliative care services provided to meet person-specific needs at the appropriate time in the preferred setting are essential supports for persons with a life limiting illness who prefer a home death. Community based palliative home care has been found to have a positive impact on 
quality of life and reduce health care expenditures for persons faced with a life limiting illness and their informal support network [3,4]. Palliative home care programs have also been shown to improve quality of life, reduce physical symptoms, reduce psychological distress, and improve accessibility to formal care providers [5]. Palliative care promotes person-specific care where resources and supports are tailored to meet need on a case-by-case basis.

Standardized assessment tools are crucial to identifying the specific needs of the person. Further, by collecting a substantial number of assessments an evidence base can be developed that can be used to design appropriate approaches to care. To this end, the interRAI Palliative Care (interRAI PC) assessment instrument was developed as part of an integrated suite of instruments spanning the continuum of care [6,7]. The interRAI assessment instruments use common measurements and common assessment approaches to enable linkage of individual level data as persons transition across the continuum of care (e.g., from home care and long-term care to acute and mental health services) [8-10].

The interRAI PC is a standardized comprehensive assessment tool providing person-specific information to inform the care planning process. Information gathered from the interRAI PC enables assessment of outcomes, tracking of change in person needs over time, quality assessment, and may inform future development of a case mix system for persons receiving palliative care [6]. In Canada, the interRAI suite of assessment instruments are used in multiple care settings including: home care, assisted living, complex continuing care (CCC), LTC, acute care, inpatient and community mental health, and post-acute rehabilitation [11,12]. The interRAI PC has been newly implemented in Ontario, Canada, joining other mandated interRAI instruments including the RAIHome Care, RAI-Mental Health, interRAI Community Health Assessment, interRAI Contact Assessment and RAI 2.0 [11-13].

The interRAI PC is used by front line palliative care providers as they seek to address multiple aspects of clinical complications, physical and cognitive decline, as well as social support systems and end of life preferences. The communication of this information between the person and provider of care is geared to ensuring that the unique needs of the person are addressed in an appropriate and timely manner.

The interRAI PC Clinical Assessment Protocols (CAPs) focus on specific clinical, functional, and life quality issues [7]. Using algorithms embedded within the interRAI PC, the CAPs alert the assessor to specific problems and indicate either risk of their appearance or potential for improvement, if present. Both can be addressed in the care plan [14]. Each CAP contains four components: issue statement, goals of care, triggers, and guidelines. The issue statement provides a clear rationale for why the specific CAP domain should be an important part of the palliative care services under consideration and examines the impact of the clinical issue on the person's life. The goals of care highlight the benefits of potential intervention [15-18]. These vary by CAP and may include: reducing distress, resolving the problem in its entirety, reducing the risk of deterioration, eliminating side effects of an intervention, or increasing the opportunity to improve or maintain function when possible. Targeting triggers (also embedded within the instrument) have been created based on a large palliative care dataset to identify which persons appear to be most likely to benefit from an intervention.

Detailed technical information on the statistical code for the CAP triggers may be accessed via www.interRAI. org. Best practice care guidelines summarize what are likely the most appropriate responses to the issue. By outlining various approaches to the problem, clinicians are able to consider underlying issues and treatment alternatives when creating a person-specific plan of care. The CAP manual includes additional resources and reference materials enabling quick access to more detailed information.

The first set of eight interRAI PC CAPs released in 2013 address the following domains: Fatigue, Sleep Disturbance, Nutrition, Pressure Ulcers, Pain, Dyspnea, Mood Disturbance, and Delirium [7]. These CAPs are distinct from other CAPs, such as those accompanying the interRAI home care, long-term care, and mental health instruments. These CAPs allow for prioritizing the person's needs as death approaches. The CAPs highlight areas of need that may benefit from treatment or targeted care, even in the final stages of life. Previously, benefits of the CAPs for both risk assessment and care planning in the community and in institutional mental health settings have been documented $[19,20]$. Moreover, the benefits for clinicians to use the CAPs to assist in identifying at-risk residents residing in long-term care facilities has also been highlighted [21].

This paper provides the first description of the strengths and limitations of the interRAI PC Clinical Assessment Protocols (CAPs). An overview of the CAP development process will be provided, as well as an examination of how the CAPs function in relation to physical, psychological, and social characteristics.

\section{Methods}

\section{CAP development process}

CAP development entailed a three phase multi-year process conducted by an international committee with members from nine countries. The committee considered evidence from peer-reviewed literature and international best practice guidelines [7]. Phase one focused on a review 
of best practice guidelines for each CAP domain area, gathered from at least three global regions. The focus of the CAP domain was then identified. When guidelines were unavailable, relevant peer-reviewed publications were reviewed. During phase two, consultation with subjectmatter experts from around the world was undertaken. Direct evaluation of the CAPs by palliative care providers was conducted to support face validity. Responses from both interRAI and outside experts supported the premise that each CAP captured accurate and clinically relevant information. Recommendations during this consultation process were incorporated into the CAP frameworks. Phase three focused on creation of triggering algorithms based on an analysis of Canadian data. The interRAI PC CAP manual was developed, detailing information on trigger rates, factors associated with triggering, and best practice guidelines [7].

\section{Data source}

The interRAI PC assessment instrument includes more than 280 items, covering 75 key areas, grouped into 17 specific domains including demographic and intake information, medical diagnoses and conditions, physical and cognitive functioning, and psycho-social and emotional wellbeing $[6,7]$. The instrument helps clinicians to provide person-level care rather than site-specific care and therefore may be employed in multiple care settings such as community-based, hospice, or residential care facilities. Assessments, completed by trained individuals with professional backgrounds, including nursing and social work, consolidate information from direct observation, medical records, and communication with the person, their health team, and their informal support network. Information gathered from the interRAI PC may assist the person and members of their care team, in partnership, to identify, address, and evaluate personspecific care needs. The breadth of information collected provides a comprehensive description of the person. Items contained in the interRAI PC have shown excellent interrater and test-retest reliability $[6,19]$. Data gathered from interRAI PC assessments provide an evidence base, which when combined with clinical judgment, is useful to inform the development and implementation of care plans tailored to the unique needs of each person.

\section{Clinical assessment protocols (CAPs)}

The Dyspnea CAP and Delirium CAP both have binary triggers (do not trigger/trigger) in comparison to the other six CAPs that have two triggering levels (do not trigger/trigger level one/trigger level two). The Dyspnea CAP identifies persons experiencing shortness of breath and highlights strategies to recognize the onset and severity of symptoms [22]. Persons who are currently experiencing delirium trigger the Delirium CAP that highlights clinical strategies not only to identify and treat symptoms but also to prevent foreseeable complications and to improve QOL [23]. The Fatigue CAP is the most frequently triggered CAP. It differentiates the risk for persons currently or at risk of experiencing fatigue (medium risktrigger level 1, high risk-trigger level 2) and outlines key considerations to address both causes and symptom reduction [24]. Based on the interRAI Pain Scale, the Pain CAP prioritizes persons experiencing pain (medium-trigger level 1, high-trigger level 2) and provides best practice guidelines for assessment and management strategies [25]. The Mood Disturbance CAP differentiates levels of risk of depression by symptom frequency (single-trigger level 1 , multiple-trigger level 2) with a goal to improve psychological well-being [26]. It outlines best practice approaches that address the symptoms and investigate the type of disorder. It then lists key considerations for potential treatment and monitoring of the disorder. The Sleep Disturbance CAP differentiates the potential to improve (moderate-trigger level 1 or high-trigger level 2) among persons experiencing a sleep disturbance. Based upon the presence of a list of reversible issues, the Sleep Disturbance CAP focuses on strategies to reduce the disturbance, increase comfort, and improve functioning [27]. The $\mathrm{Nu}-$ trition CAP identifies persons who may benefit from education and interventions to optimize energy and protein intake, reduce anxiety about not eating, or who could benefit from interventions addressing hunger [28]. Trigger levels focus on persons with a low body mass index (BMI) and differentiates levels based on absence (trigger level 1) or presence (trigger level 2) of weight loss. The Pressure Ulcers CAP emphasizes the importance of appropriate treatment and identifies potential for improvement (moderate-trigger level 1, high-trigger level 2) for persons with pressure ulcers [29].

\section{Study sample}

De-identified cross-sectional pilot data from 6,769 interRAI PC assessments gathered between 2006 and 2011 from palliative home care clients in Ontario, Canada were included for analysis. When follow-up assessments were available, only the first assessment was included. Data were collected from six Community Care Access Centre's (CCAC), which serve as the first point of access to community based care across the province. Each CCAC is tasked to coordinate specialized supports, including palliative care, for persons under its jurisdiction and to connect persons requiring care with available services/ resources in the person's home or within the respected community. The six CCAC pilot sites were located across various geographical regions of Ontario from north to south, east to west and ranged from primarily metropolitan urban to more rural and geographically dispersed districts. Sample characteristics are shown in Table 1. Age 
Table 1 Sample characteristics of clients receiving palliative home care services 2006-2011, Ontario, Canada $(\mathrm{N}=6,769)$

Total population \% (N)

\begin{tabular}{|c|c|}
\hline \multicolumn{2}{|l|}{ Age groups } \\
\hline $18-44$ & $4.3(288)$ \\
\hline $45-64$ & $29.9(2,025)$ \\
\hline $65-74$ & $25.4(1,718)$ \\
\hline $75-84$ & $28.7(1,943)$ \\
\hline $85+$ & $11.7(795)$ \\
\hline \multicolumn{2}{|l|}{ Gender } \\
\hline Male & $49.1(3,303)$ \\
\hline Female & $50.9(3,418)$ \\
\hline \multicolumn{2}{|l|}{ Estimated prognosis } \\
\hline Greater than 6 months & $41.5(2,310)$ \\
\hline 6 weeks to 6 months & $48.1(2,677)$ \\
\hline Less than 6 weeks & $8.4(468)$ \\
\hline Death Imminent & $2.0(110)$ \\
\hline \multicolumn{2}{|l|}{ CCAC Site location } \\
\hline Site 1 & $4.0(270)$ \\
\hline Site 2 & $47.7(4,581)$ \\
\hline Site 3 & $14.6(991)$ \\
\hline Site 4 & $7.5(510)$ \\
\hline Site 5 & $2.1(142)$ \\
\hline Site 6 & $4.1(275)$ \\
\hline \multicolumn{2}{|l|}{ Diagnosis } \\
\hline Have cancer diagnosis & $86.8(5,875)$ \\
\hline Metastatic & $40.0(2,710)$ \\
\hline Not Metastatic & $46.8(3,165)$ \\
\hline Do not have cancer & $9.8(666)$ \\
\hline Diagnosis unspecified & $3.4(228)$ \\
\hline
\end{tabular}

ranged from 18 to 107 years with a mean age of 70.0 years (SD \pm 13.4 years), of whom more than $80 \%$ reported a diagnosis of cancer $(n=5,875)$. The majority of persons were rated by clinicians to have an estimated prognosis of greater than 6 weeks, with more $40 \%(n=2,310)$ having an estimated prognosis of greater than six months at the time of the assessment. Only $2 \%(n=110)$ had a prognosis of death being imminent.

\section{Analysis}

Univariate distributional properties were examined for all eight interRAI PC CAPs and cross tabulations were used to examine the hierarchical triggering structure of the CAPs. Associated covariates including age, gender, estimated prognosis, geographic location, and disease diagnosis, were examined using chi-square to determine significant relationships. The hierarchical analysis also employed chi-square analysis to examine covariates among CAPs. All analyses were performed using SAS Version 9.2 with an alpha level of $\mathrm{p}<0.05$ for all statistical tests; however, the sample is sufficiently large that in some cases differences may be statistically significant at the level, but of modest clinical importance.

Informed consent was not required for the interRAI PC assessment process because the assessment was used as part of the standard of care in routine clinical practice. The interRAI PC data were deidentified prior to submission to the University of Waterloo in order to ensure that they did not constitute personally identifiable health records. Ethics clearance for the analyses of these secondary data was obtained through the University of Waterloo's Office of Research Ethics (\#19424).

\section{Results}

Each CAP contains individualized triggers occurring at different rates from 74\% (Fatigue CAP) to less than 15\% (Delirium and Pressure Ulcers CAPs) (Figure 1). Spearman's

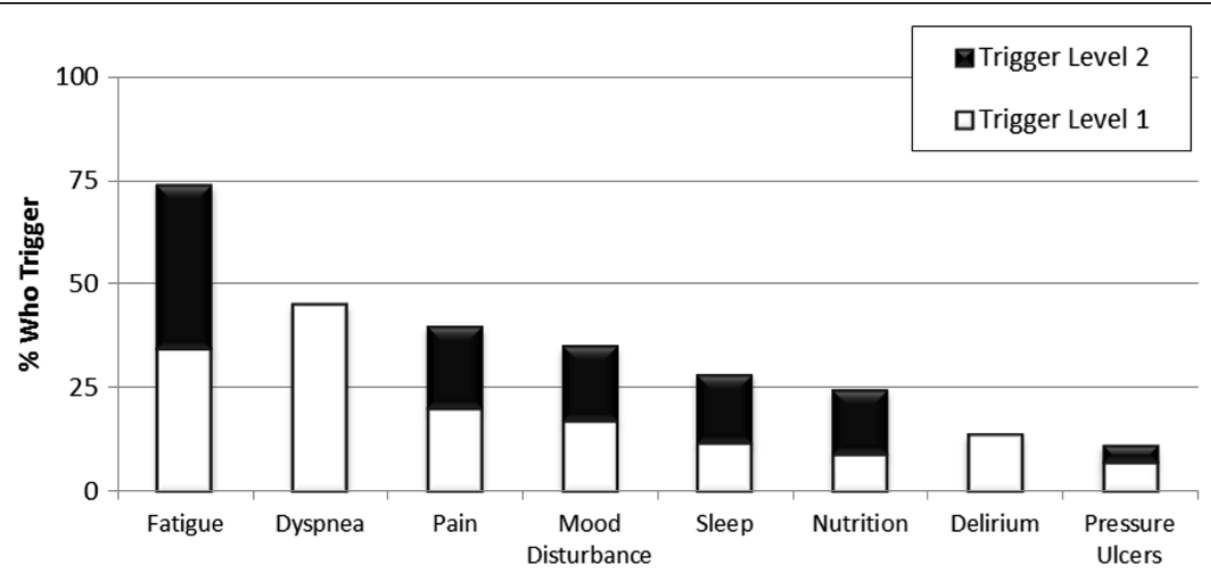

Figure 1 Triggering rates by CAP of clients receiving palliative home care services 2006-2011, Ontario, Canada $(N=6,769)$. 
rank correlations suggested that the majority of CAPs were reasonably independent from each other. Modest correlations were evident between the Fatigue and Delirium CAPs (0.20) and Fatigue and Mood Disturbance CAPs (0.26). Nearly 9 in 10 persons triggered at least one CAP (87.9\%, $\mathrm{n}=5,950$ ) and approximately two thirds triggered more than two CAPs (Figure 2). Variable distribution differed across the four CAPs dealing with clinical complexity: Dyspnea, Nutrition, Pain, and Pressure Ulcers (Tables 2 and 3), the CAPs dealing with performance: Fatigue and Sleep Disturbance (Table 4), and the cognition/mental health CAPs: Delirium and Mood Disturbance (Table 5). Triggering rates differed by CAP and by geographic location.

The prevalence of persons who triggered the Dyspnea CAP generally increased with age, increased as the estimated prognosis was shorter, and was higher in females. In addition, persons with a non-cancer diagnosis were significantly more likely to trigger the Dyspnea CAP compared to persons with a cancer diagnosis $(62.3 \%$ vs. $42.2 \% \mathrm{p}<0.0001)$. CCAC Site 5 reported substantially lower rates of persons who triggered the Dyspnea CAP than other CCAC sites (23.6\% for CCAC Site 1 vs. range from $37.5 \%$ in CCAC Site 1 to $46.87 \%$ in CCAC Site 2).

The prevalence of persons who triggered the Nutrition CAP also increased as the estimated prognosis was shorter and was more common in females. An exception to the overall increase in triggering of the Nutrition CAP with increased age overall was, a curvilinear relationship observed among persons who triggered level one (low BMI) of the Nutrition CAP where the youngest (aged 18-44) and the oldest old (85+) age groups were more likely to trigger. For the Nutrition CAP, although overall triggering prevalence's were comparable across sites, persons from the CCAC Site 5 reported the highest triggering rates at level one (17.5\%) but the lowest prevalence at level two (9.7\%). In contrast, CCAC Site 1 exhibited the lowest triggering rates for the Nutrition
CAP at level one $(4.7 \%)$ and the highest prevalence of Nutrition CAP triggering rates at level two (21.1\%).

The prevalence of persons who triggered the Pain CAP was highest for persons aged 18-44 and generally decreased with age. Prevalence of triggering the Pain CAP increased as the estimated prognosis was shorter. No differences were observed in prevalence of Pain CAP triggering by gender. Persons with a cancer diagnosis were significantly more likely to trigger the Pain CAP compared to persons with a non-cancer diagnosis $(42.1 \%$ vs. 26.8\% $\mathrm{p}<0.0001)$. Variation in Pain CAP triggering ranged substantially from a low of $22.9 \%$ in CCAC Site 3 to more than double that in CCAC Site 4 (51.1\%).

Persons over the age of 65 exhibited were more likely to trigger the Pressure Ulcers CAP and more specifically, most likely to trigger at level 1 (Difficult to improve). No differences were observed in prevalence of Pressure Ulcers CAP triggering by gender. Persons with a non-cancer diagnosis were significantly more likely to trigger the Pressure Ulcers CAP compared to persons with a cancer diagnosis $(24.4 \%$ vs. $9.0 \% \mathrm{p}<0.0001)$. Geographic variation in Pressure Ulcer CAP triggering ranged from $6.4 \%$ in CCAC Site 6 to $12.5 \%$ in CCAC Site 3 .

The prevalence of persons who triggered the Fatigue CAP increased with age (Table 4). It also increased as the estimated prognosis was shorter from $54.4 \%$ among those with a prognosis greater than 6 months to a prevalence of $97.5 \%$ for those whose death was imminent. The number of persons who triggered the Fatigue CAP at level two (high risk) nearly tripled from $26.6 \%$ for those with an estimated prognosis of greater than six months to $88.6 \%$ when death is imminent. No significant differences were evident by gender. Persons with a noncancer diagnosis were significantly more likely to trigger the Fatigue CAP compared to persons with a cancer diagnosis $(82.8 \% \%$ vs. $71.9 \% \mathrm{p}<0.0001)$. Prevalence of Fatigue CAP triggering ranged greatly by site where

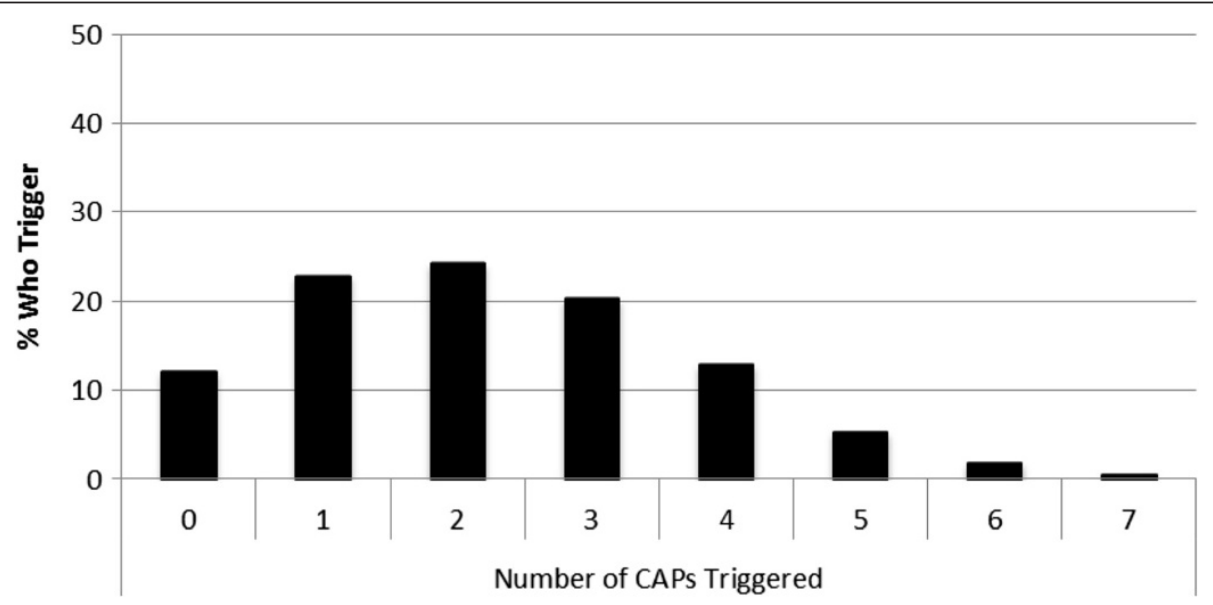

Figure 2 Number of CAPs triggered by clients receiving palliative home care services 2006-2011, Ontario, Canada ( $N=6,769$ ). 
Table 2 Distribution of background characteristics by dyspnea and nutrition clinical complexity CAPs of clients receiving palliative home care services 2006-2011, Ontario, Canada $(N=6,769)$

\begin{tabular}{|c|c|c|c|c|c|c|c|c|c|}
\hline & \multicolumn{4}{|c|}{ Dyspnea } & \multicolumn{5}{|c|}{ Nutrition } \\
\hline & $\begin{array}{l}\text { Not triggered } \\
\%(\mathrm{~N})\end{array}$ & $\begin{array}{l}\text { Trigger level } \\
1 \%(\mathrm{~N})\end{array}$ & $\begin{array}{l}\text { Chi-square } \\
\text { (df) }\end{array}$ & p Value & $\begin{array}{l}\text { Not triggered } \\
\%(\mathrm{~N})\end{array}$ & $\begin{array}{l}\text { Trigger level } \\
1 \%(\mathrm{~N})\end{array}$ & $\begin{array}{l}\text { Trigger level } \\
2 \%(\mathrm{~N})\end{array}$ & $\begin{array}{l}\text { Chi-square } \\
\text { (df) }\end{array}$ & p Value \\
\hline \multicolumn{10}{|l|}{ Age groups } \\
\hline $18-44$ & $68.6(192)$ & $31.4(88)$ & $31.8(4)$ & $<.0001$ & $76.4(155)$ & $12.3(25)$ & $11.3(23)$ & $28.4(8)$ & 0.0004 \\
\hline $45-64$ & $57.1(1,140)$ & 42.9 (858) & & & $78.7(1,044)$ & $6.3(84)$ & 14.9 (198) & & \\
\hline $65-74$ & 54.4 (919) & $45.6(769)$ & & & 76.5 (855) & $8.4(94)$ & $15.1(169)$ & & \\
\hline $75-84$ & $52.0(994)$ & 48.0 (919) & & & $73.1(882)$ & $10.3(124)$ & $16.7(201)$ & & \\
\hline $85+$ & $54.0(420)$ & $45.9(356)$ & & & $71.9(330)$ & $12.2(56)$ & $15.9(73)$ & & \\
\hline \multicolumn{10}{|l|}{ Gender } \\
\hline Male & $53.1(1,727)$ & $46.9(1,526)$ & $9.6(1)$ & 0.002 & $78.9(1,645)$ & $6.3(132)$ & $14.8(308)$ & $38.1(2)$ & $<.0001$ \\
\hline Female & $56.9(1,908)$ & $43.1(1,446)$ & & & $72.5(1,591)$ & $11.4(251)$ & $16.1(353)$ & & \\
\hline \multicolumn{10}{|l|}{ Estimated prognosis } \\
\hline Death imminent & $37.4(40)$ & $62.6(67)$ & $105.2(3)$ & $<.0001$ & $64.7(33)$ & $2.0(1)$ & $33.3(17)$ & $78.4(6)$ & $<.0001$ \\
\hline Less than 6 weeks & $43.8(203)$ & $56.3(261)$ & & & $65.2(193)$ & $6.8(20)$ & $28.0(83)$ & & \\
\hline 6 weeks to 6 months & $53.4(1,413)$ & $46.6(1,235)$ & & & $73.5(1,407)$ & $9.2(176)$ & $17.3(331)$ & & \\
\hline Greater than 6 months & $63.7(1,454)$ & $36.3(829)$ & & & $79.4(1,355)$ & $9.4(161)$ & $11.2(191)$ & & \\
\hline \multicolumn{10}{|l|}{ Geographic location } \\
\hline Site 1 & $62.6(167)$ & $37.5(100)$ & $41.5(5)$ & $<.0001$ & $74.1(172)$ & $4.7(11)$ & $21.1(49)$ & $38.9(10)$ & $<.0001$ \\
\hline Site 2 & $53.3(2,389)$ & $46.7(2,091)$ & & & $77.1(1,945)$ & $8.2(208)$ & $14.7(371)$ & & \\
\hline Site 3 & $58.1(574)$ & $41.9(414)$ & & & $71.3(627)$ & $10.8(95)$ & $17.9(157)$ & & \\
\hline Site 4 & $54.0(273)$ & $46.1(233)$ & & & $75.6(272)$ & $10.8(39)$ & $13.6(49)$ & & \\
\hline Site 5 & $76.4(107)$ & $23.6(33)$ & & & $72.8(75)$ & $17.5(18)$ & $9.7(10)$ & & \\
\hline Site 6 & $56.6(155)$ & $43.4(119)$ & & & $81.4(175)$ & $5.6(12)$ & $13.0(28)$ & & \\
\hline \multicolumn{10}{|l|}{ Diagnosis } \\
\hline Have cancer diagnosis & $57.8(2,295)$ & $42.2(1,678)$ & 98.9 (3) & $<.0001$ & $76.1(1,912)$ & $8.71(219)$ & $15.2(383)$ & $39.5(6)$ & $<.0001$ \\
\hline $\begin{array}{l}\text { Have cancer and } \\
\text { non-cancer diagnosis }\end{array}$ & $56.5(1,023)$ & 43.5 (789) & & & $77.8(1,047)$ & $6.76(91)$ & $15.5(208)$ & & \\
\hline $\begin{array}{l}\text { Have non-cancer } \\
\text { diagnosis only }\end{array}$ & $37.8(245)$ & $62.3(404)$ & & & $67.5(241)$ & $16.8(60)$ & $15.7(56)$ & & \\
\hline Diagnosis unspecified & $46.2(103)$ & $53.9(119)$ & & & $68.8(66)$ & $13.5(13)$ & $17.7(17)$ & & \\
\hline
\end{tabular}

Note: df denotes degrees of freedom.

CCAC Site 2 reported the lowest rates for Fatigue CAP triggering (71.9\%) compared to a high of $83.8 \%$ reported by CCAC Site 4 .

The prevalence of persons who triggered the Sleep Disturbance CAP increased with age (Table 4). While similar rates were observed by age for triggering the Sleep Disturbance CAP at level one (moderate potential to improve), $25.1 \%$ of younger persons (aged 18-44) reported triggering the Sleep Disturbance CAP at level two (high potential to improve) double that reported by the oldest old (12.4\%). Prevalence of triggering the Sleep Disturbance CAP was higher for males, persons with a shorter estimated prognosis, and persons with both a cancer and non-cancer diagnosis. Geographic variation in Sleep Disturbance CAP triggering rates ranged by CCAC site from $31.2 \%$ in CCAC Site 3 to low of $23.5 \%$ in CCAC Site 1.

A general increase in Delirium CAP triggering is shown by age. Persons aged 85 or greater exhibited the highest triggering rate, nearly double the rate of those aged 18-44. The Delirium CAP is most commonly triggered by persons with a shorter prognosis, with over two-thirds triggering the Delirium CAP when death is imminent. Those with a non-cancer diagnosis were more likely to trigger the Delirium CAP than those with cancer. Site variations in Delirium CAP triggering ranged greatly from $6.4 \%$ in the CCAC Site $5(\mathrm{n}=9)$ to over $20 \%$ in CCAC Site $1(n=55)$.

Prevalence of Mood Disturbance CAP triggering frequency decreased with age with the exception for youngest 
Table 3 Distribution of background characteristics by pain and pressure ulcers clinical complexity CAPs of clients receiving palliative home care services 2006-2011, Ontario, Canada $(N=6,769)$

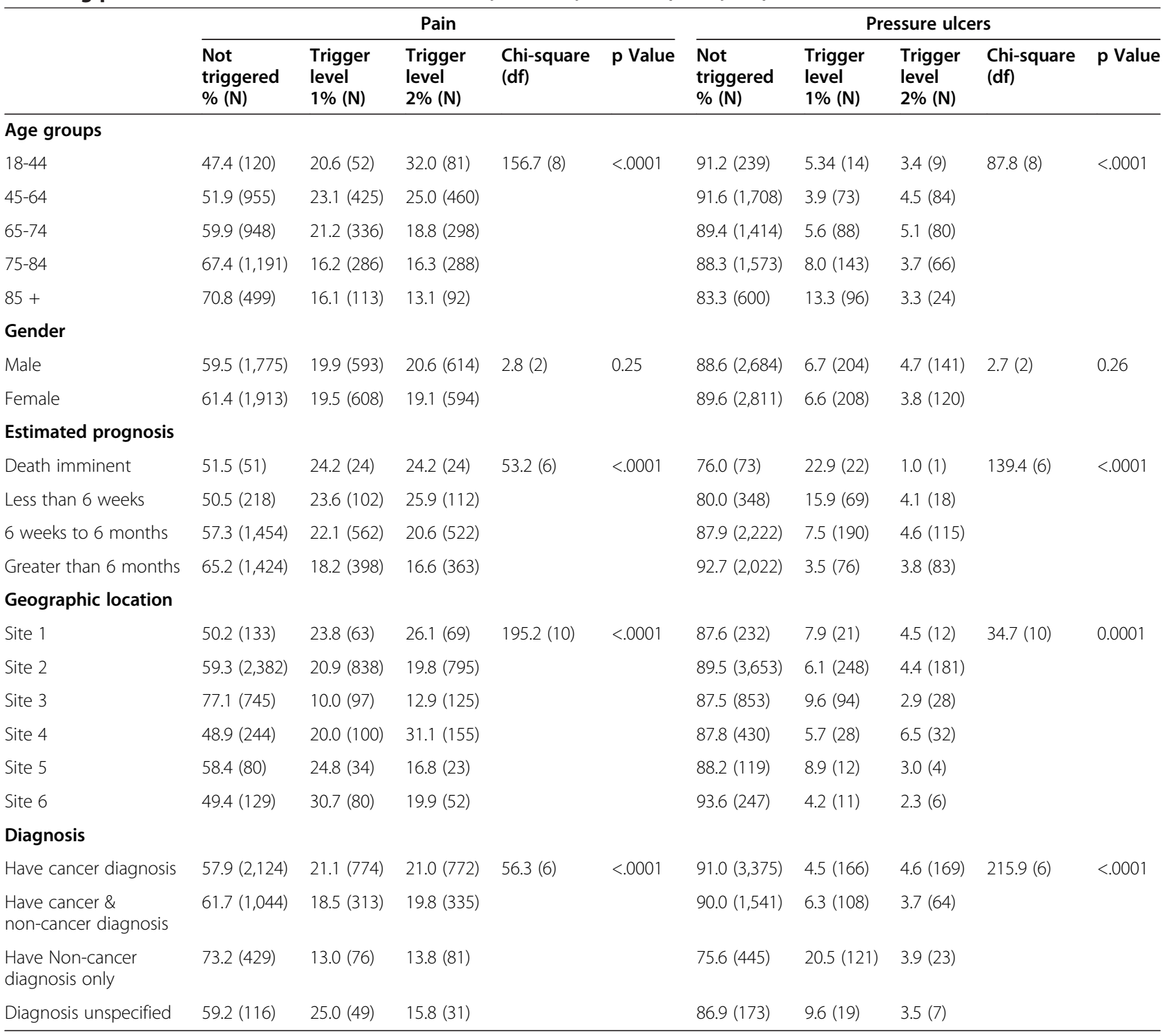

Note: df denotes degrees of freedom.

age group. However, among those who triggered the Mood Disturbance CAP, younger persons were more likely to trigger at a level two. The Mood Disturbance CAP was most commonly triggered by persons with an estimated prognosis of less than 6 weeks. Variation in Mood Disturbance CAP triggering rates was less than $8 \%$ between CCACs $(59.0 \%$ in CCAC Site 6 to $66.9 \%$ in CCAC Site 2). Persons with a cancer and non-cancer diagnoses triggered the Mood Disturbance CAP more frequently than those with only a cancer or only a non-cancer diagnosis.

Through examination of the count of triggered CAPs a hierarchical structure in triggering emerged (Figure 3). Fatigue was the most commonly triggered CAP, triggered by $38.9 \%$ of persons who triggered only one CAP to over
90\% of persons who triggered three to five CAPs and $100 \%$ of persons to triggered six or more CAPs. Captured in the percentage of persons who trigger only one CAP, the Fatigue CAP, Pain CAP, Nutrition CAP, and Dyspnea CAP emerged as early-triggered CAPs. In contrast, the Delirium and Pressure Ulcer CAPs emerged as latetriggered CAPs. Consequently, persons who triggered only one CAP, were most likely to trigger the Fatigue, Pain, Nutrition, or Dyspnea CAPs and were least likely to trigger the Delirium CAP and Pressure Ulcer CAP. Among those who triggered seven CAPs, all persons triggered the Fatigue and Mood Disturbance CAPs and over 90\% triggered the Dyspnea, Nutrition, and Pain CAPs. In contrast, the Sleep Disturbance, Delirium, and Pressure Ulcers CAPs 
Table 4 Distribution of background characteristics by fatigue and sleep disturbance performance CAPs of clients receiving palliative home care services 2006-2011, Ontario, Canada $(N=6,769)$

\begin{tabular}{|c|c|c|c|c|c|c|c|c|c|c|}
\hline & \multicolumn{5}{|c|}{ Fatigue } & \multicolumn{5}{|c|}{ Sleep disturbance } \\
\hline & $\begin{array}{l}\text { Not } \\
\text { triggered } \\
\%(\mathrm{~N})\end{array}$ & $\begin{array}{l}\text { Trigger } \\
\text { level } \\
1 \%(\mathrm{~N})\end{array}$ & $\begin{array}{l}\text { Trigger } \\
\text { level } \\
2 \%(\mathrm{~N})\end{array}$ & $\begin{array}{l}\text { Chi-square } \\
\text { (df) }\end{array}$ & p Value & $\begin{array}{l}\text { Not } \\
\text { triggered } \\
\%(\mathrm{~N})\end{array}$ & $\begin{array}{l}\text { Trigger } \\
\text { level } \\
1 \%(\mathrm{~N})\end{array}$ & $\begin{array}{l}\text { Trigger } \\
\text { level } \\
2 \%(\mathrm{~N})\end{array}$ & $\begin{array}{l}\text { Chi-square } \\
\text { (df) }\end{array}$ & p Value \\
\hline \multicolumn{11}{|l|}{ Age groups } \\
\hline $18-44$ & $31(62)$ & $40.0(80)$ & $29.0(58)$ & $23.2(8)$ & 0.003 & $62.8(135)$ & $12.1(26)$ & $25.1(54)$ & $74.7(8)$ & $<.0001$ \\
\hline $45-64$ & $26.3(396)$ & $36.3(547)$ & $37.4(564)$ & & & $66.2(1,055)$ & $12.1(192)$ & $21.7(346)$ & & \\
\hline $65-74$ & $27.5(351)$ & $31.8(406)$ & $40.7(520)$ & & & $73.6(1,015)$ & $11.5(159)$ & $14.9(206)$ & & \\
\hline $75-84$ & $25.7(364)$ & $34.4(487)$ & $39.9(565)$ & & & $76.8(1,187)$ & $10.0(154)$ & $13.3(205)$ & & \\
\hline $85+$ & $24.5(136)$ & $30.6(170)$ & 44.9 (249) & & & $74.5(462)$ & $13.1(81)$ & $12.4(77)$ & & \\
\hline \multicolumn{11}{|l|}{ Gender } \\
\hline Male & $25.3(607)$ & 33.9 (815) & 40.9 (982) & $4.7(2)$ & 0.09 & $69.5(1,813)$ & $13.3(346)$ & $17.2(449)$ & $20.2(2)$ & $<.0001$ \\
\hline Female & $27.6(693)$ & $34.2(860)$ & $38.2(960)$ & & & $74.3(2,014)$ & $9.7(263)$ & $16.0(433)$ & & \\
\hline \multicolumn{11}{|l|}{ Estimated prognosis } \\
\hline Death imminent & $2.5(2)$ & $8.9(7)$ & $88.6(70)$ & $856.8(6)$ & $<.0001$ & $68.8(53)$ & $9.1(7)$ & $22.1(17)$ & $23.1(6)$ & 0.0008 \\
\hline Less than 6 weeks & $3.8(15)$ & $25.3(99)$ & $70.8(277)$ & & & $75.3(289)$ & $5.0(19)$ & $19.8(76)$ & & \\
\hline 6 weeks to 6 months & $14.1(337)$ & $41.9(1,000)$ & $44.0(1,052)$ & & & $71.5(1,635)$ & $11.2(257)$ & $17.3(394)$ & & \\
\hline Greater than 6 months & $45.6(955)$ & $27.9(584)$ & $26.6(557)$ & & & $72.0(1,483)$ & $12.5(258)$ & $15.5(320)$ & & \\
\hline \multicolumn{11}{|l|}{ Geographic location } \\
\hline Site 1 & $19.5(50)$ & $35.2(90)$ & $45.3(116)$ & $202.3(10)$ & $<.0001$ & 76.5 (199) & $6.9(18)$ & $16.5(43)$ & $31.6(10)$ & 0.0005 \\
\hline Site 2 & $28.9(868)$ & $28.7(860)$ & $42.4(1,273)$ & & & $73.1(2,499)$ & $11.5(393)$ & $15.5(529)$ & & \\
\hline Site 3 & $28.5(262)$ & $45.1(415)$ & $26.5(244)$ & & & $67.1(633)$ & $13.8(130)$ & $19.09(180)$ & & \\
\hline Site 4 & $15.8(70)$ & $40.3(178)$ & 43.9 (194) & & & $70.8(312)$ & $7.9(35)$ & $21.32(94)$ & & \\
\hline Site 5 & $18.0(20)$ & $64.0(71)$ & $18.0(20)$ & & & $73.9(68)$ & $12.0(11)$ & $14.13(13)$ & & \\
\hline Site 6 & $17.4(39)$ & $33.9(76)$ & $48.7(109)$ & & & $72.6(143)$ & $12.7(25)$ & $14.72(29)$ & & \\
\hline \multicolumn{11}{|l|}{ Diagnosis } \\
\hline Have cancer diagnosis & $28.1(823)$ & $34.7(1,016)$ & $37.2(1,090)$ & $94.2(6)$ & $<.0001$ & $73.6(2,360)$ & $11.3(362)$ & $15.1(484)$ & $19.8(6)$ & 0.003 \\
\hline $\begin{array}{l}\text { Have cancer and } \\
\text { non-cancer diagnosis }\end{array}$ & $26.2(375)$ & $37.1(530)$ & $36.7(525)$ & & & $68.8(1,038)$ & $11.9(180)$ & $19.28(291)$ & & \\
\hline $\begin{array}{l}\text { Have non-cancer } \\
\text { diagnosis only }\end{array}$ & $17.2(79)$ & $24.8(114)$ & $58.0(266)$ & & & $71.2(349)$ & $9.8(48)$ & $18.98(93)$ & & \\
\hline Diagnosis unspecified & $23.4(32)$ & $21.9(30)$ & $54.7(75)$ & & & 71.8 (107) & $14.8(22)$ & $13.4(20)$ & & \\
\hline
\end{tabular}

Note: df denotes degrees of freedom.

were triggered less frequently. When all but one CAP were triggered, the Sleep Disturbance, Delirium, and Pressure Ulcers CAPs remained least likely to be triggered.

\section{Discussion}

Analyses of interRAI PC CAP triggering rates in Ontario, Canada illustrate the information to be gained from the interRAI PC, a comprehensive standardized assessment instrument. Covering especially pertinent clinical issues, performance, and mental health/cognition domains, the eight interRAI PC CAPs emphasize need for care planning in key areas of palliative care. The majority of persons triggered two or more CAPs reflecting high levels of clinical need within the palliative home care sample. Variation in
CAP triggering was evident based on the age, estimated prognosis, geographic location and diagnosis of the person. Older persons and those with a shorter estimated prognosis were most likely to trigger multiple CAPs.

As emphasized by the WHO, comprehensive assessment is an integral component of quality palliative care. White, McMullan, and Doyle found that two thirds of symptoms experienced by persons receiving palliative care services were not immediately self-reported [30]. Instead, the majority of symptoms were detected through systematic questioning during assessment. As fatigue and dyspnea emerged as early-triggering CAPs, it is suggested that systematic questioning of these symptoms be prioritized during all clinical assessments. Treatment 
Table 5 Distribution of background characteristics by delirium and mood disturbance cognition/mental health CAPs of clients receiving palliative home care services 2006-2011, Ontario, Canada $(N=6,769)$.

\begin{tabular}{|c|c|c|c|c|c|c|c|c|c|}
\hline & \multicolumn{4}{|c|}{ Delirium } & \multicolumn{5}{|c|}{ Mood disturbance } \\
\hline & $\begin{array}{c}\text { Not } \\
\text { triggered } \\
\%(\mathrm{~N})\end{array}$ & $\begin{array}{c}\text { Trigger } \\
\text { level } \\
1 \%(\mathrm{~N})\end{array}$ & $\begin{array}{l}\text { Chi-square } \\
\text { (df) }\end{array}$ & p Value & $\begin{array}{c}\text { Not } \\
\text { triggered } \\
\%(\mathrm{~N})\end{array}$ & $\begin{array}{c}\text { Trigger } \\
\text { level } \\
1 \%(\mathrm{~N})\end{array}$ & $\begin{array}{c}\text { Trigger } \\
\text { level } \\
2 \%(\mathrm{~N})\end{array}$ & $\begin{array}{l}\text { Chi-square } \\
\text { (df) }\end{array}$ & $\mathrm{p}$ Value \\
\hline \multicolumn{10}{|l|}{ Age groups } \\
\hline $18-44$ & $91.3(240)$ & $8.8(23)$ & $12.9(8)$ & 0.02 & $63.4(156)$ & $14.2(35)$ & $22.4(55)$ & $45.2(8)$ & $<.0001$ \\
\hline $45-64$ & $86.8(1,601)$ & $13.2(244)$ & & & $61.4(1,105)$ & $17.5(315)$ & $21.2(381)$ & & \\
\hline $65-74$ & $86.1(1,348)$ & $13.9(217)$ & & & $63.8(953)$ & $18.0(269)$ & $18.2(272)$ & & \\
\hline $75-84$ & $87.1(1,540)$ & $12.9(228)$ & & & $68.1(1,161)$ & $16.2(276)$ & $15.7(267)$ & & \\
\hline $85+$ & $83.1(580)$ & $16.9(118)$ & & & $71.7(492)$ & $16.0(110)$ & $12.2(84)$ & & \\
\hline \multicolumn{10}{|l|}{ Gender } \\
\hline Male & $86.0(2,567)$ & $14.0(417)$ & $1.3(2)$ & 0.25 & $66.1(1,904)$ & 16.6 (479) & $17.2(496)$ & $2.2(2)$ & 0.33 \\
\hline Female & $87.0(2,706)$ & $13.0(403)$ & & & $64.3(1,933)$ & $17.4(522)$ & $18.3(551)$ & & \\
\hline \multicolumn{10}{|l|}{ Estimated prognosis } \\
\hline Death Imminent & $30.5(25)$ & $69.5(57)$ & $510.5(6)$ & $<.0001$ & $65.1(54)$ & $19.3(16)$ & $15.7(13)$ & $73.3(6)$ & $<.0001$ \\
\hline Less than 6 weeks & $62.1(267)$ & $37.9(163)$ & & & $51.3(205)$ & $18.5(74)$ & $30.3(121)$ & & \\
\hline 6 weeks to 6 months & $85.5(2,171)$ & $14.5(368)$ & & & $62.1(1,492)$ & $18.3(440)$ & $19.6(470)$ & & \\
\hline Greater than 6 months & $93.3(2,036)$ & $6.7(146)$ & & & $69.6(1,490)$ & $15.3(328)$ & $15.1(324)$ & & \\
\hline \multicolumn{10}{|l|}{ Geographic location } \\
\hline Site 1 & $79.6(214)$ & $20.5(55)$ & $85.1(10)$ & $<.0001$ & $62.3(167)$ & $15.3(41)$ & $22.4(60)$ & $34.9(10)$ & 0.0001 \\
\hline Site 2 & $89.1(3,560)$ & $10.9(437)$ & & & $66.9(2,666)$ & $17.2(684)$ & $16.0(638)$ & & \\
\hline Site 3 & $81.6(791)$ & 18.5 (179) & & & $62.0(591)$ & $15.3(146)$ & $22.8(217)$ & & \\
\hline Site 4 & $80.9(402)$ & $19.1(95)$ & & & $62.9(251)$ & $18.3(73)$ & $18.8(75)$ & & \\
\hline Site 5 & $93.6(131)$ & $6.4(9)$ & & & $61.1(58)$ & $17.9(17)$ & $21.1(20)$ & & \\
\hline Site 6 & $79.3(211)$ & $20.7(55)$ & & & $59.0(134)$ & $19.4(44)$ & $21.6(49)$ & & \\
\hline \multicolumn{10}{|l|}{ Diagnosis } \\
\hline Have cancer diagnosis & $88.0(3,221)$ & $12.0(438)$ & $42.5(6)$ & $<.0001$ & $66.8(2,336)$ & $16.2(566)$ & $17.0(595)$ & $31.4(6)$ & $<.0001$ \\
\hline $\begin{array}{l}\text { Have cancer and } \\
\text { non-cancer diagnosis }\end{array}$ & $86.0(1,482)$ & $14.0(241)$ & & & $60.5(1,017)$ & 18.4 (309) & $21.1(355)$ & & \\
\hline $\begin{array}{l}\text { Have non-cancer } \\
\text { diagnosis only }\end{array}$ & $78.1(449)$ & $21.9(126)$ & & & $67.5(382)$ & $18.7(106)$ & $13.8(78)$ & & \\
\hline Diagnosis unspecified & $86.3(157)$ & $13.7(25)$ & & & $70.6(132)$ & $12.8(24)$ & $16.6(31)$ & & \\
\hline
\end{tabular}

Note: df denotes degrees of freedom.

of symptoms at end of life can improve the QOL of the person and their informal support network [31]. The increased health complexity of persons requiring palliative care necessitates individualized care planning. Decision making strategies informed by evidence from the interRAI PC CAPs assist clinicians in developing a person-centered care plan, identifying areas of need, and prioritizing treatment options in consultation with the person.

Evidence of a hierarchical structure in CAP triggering may be useful to predict health complexity and change over time. For persons with multiple health concerns, the high frequency of Fatigue CAP, Dyspnea CAP, Pain CAP, Nutrition CAP, and Mood Disturbance CAP triggering warrants increased awareness on the part of the clinician and other caregivers. The Fatigue CAP and Dyspnea CAP emerge as pervasive issues among the overall palliative home care sample. When persons seem relatively stable with few major health concerns, the hierarchical nature of CAP triggering suggests clinicians should continue to investigate fatigue and dyspnea. In contrast, the Delirium CAP and Pressure Ulcers CAP trigger at higher frequency for persons nearing end of life. The Delirium CAP and Pressure Ulcers CAP, late-triggering CAPs, rarely trigger in isolation and may highlight increased client need. The late-triggering of the Delirium CAP and Pressure Ulcers CAP suggest they are indicative of later stages of need in palliative care. Further investigation into the role of CAP 


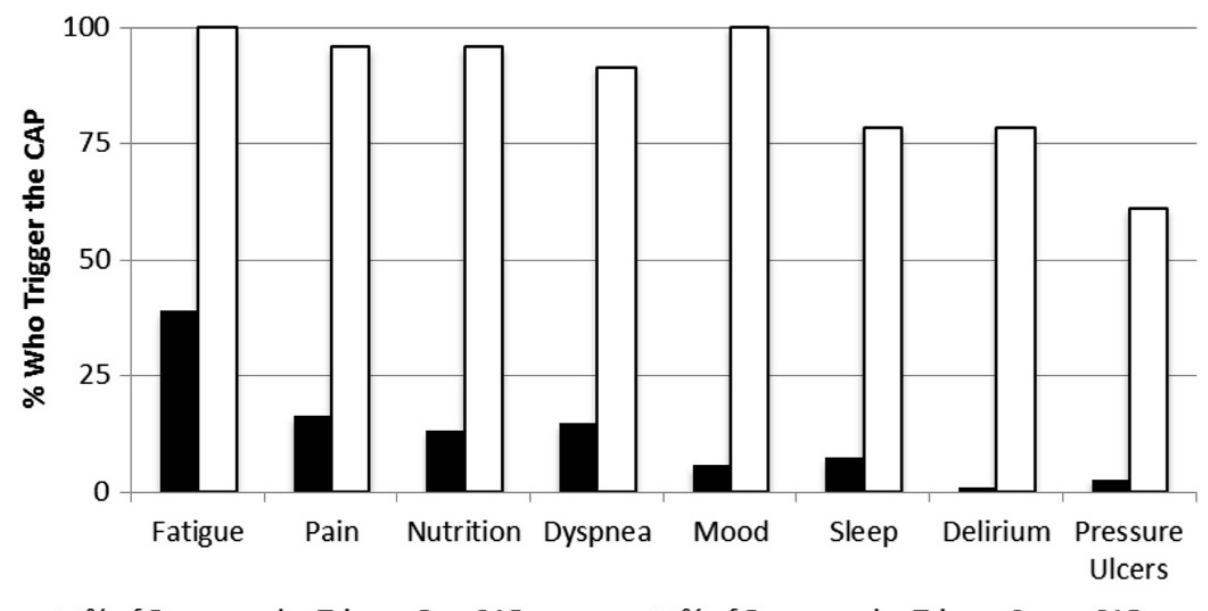

- \% of Persons who Trigger One CAP

$\square \%$ of Persons who Trigger Seven CAPs

Figure 3 CAP Triggering rates by number of CAPs triggered of palliative home care clients 2006-2011, Ontario, Canada (N=6,769).

triggering and symptom clusters is needed. The ability of the CAPs to identify symptoms also requires further assessment.

Consistent with previous research, the majority of palliative care clients in this study (86.8\%) reported a diagnosis of cancer. Seow, King and Vaitonis note that although persons with cancer receive $80-85 \%$ of palliative care services in Ontario, they account for only one third of persons who die during the time of the study [32]. Research used to inform current palliative care practice focuses almost exclusively on the needs of persons with a cancer diagnosis [33]. Benefits of palliative care for persons with a cancer diagnosis and their informal support network during the rapid decline phase preceding death are well recognized. Palliative care has been best known to benefit persons with cancer during the last few months of life $[34,35]$.

However, findings from this study stress the need to broaden understanding of how persons without cancer receiving palliative care may differ. Disease diagnosis, more specifically the presence or absence of a cancer diagnosis, was a strong predictor of health characteristics and CAP triggering among persons at the end of life. Persons reporting only a cancer diagnosis were more likely to trigger the Pain CAP. This may be expected, as there is increased awareness of the benefits of palliative care to address pain for persons with cancer and addressing uncontrolled pain is often a reason for referral. Therefore, persons with cancer who are experiencing challenges with pain may be more likely to be referred for palliative care services.

In contrast, those reporting only non-cancer diagnoses such as heart failure, stroke, COPD, or dementia, were significantly more likely to trigger the Dyspnea CAP, Nutrition CAP, Pressure Ulcers CAP, Fatigue CAP, and
Delirium CAP. This suggests that persons with noncancer diagnoses, who access palliative care services in Ontario, are more likely to exhibit increased health complexity and require person-specific tailoring of interventions to address multiple symptoms. Persons who reported both a cancer and non-cancer diagnosis were most likely to trigger the Mood Disturbance and Sleep Disturbance CAPs. For the Mood Disturbance CAP, the prevalence of triggering with a single symptom was equally as high for those with non-cancer and cancer diagnoses and for persons with non-cancer diagnosis only. However, those with both a cancer and non-cancer diagnosis were much more likely to trigger at a level 2 . The level of psychosocial distress may be comparable between persons with non-cancer and cancer diagnoses [36]; however the present results suggest this burden may be amplified when other conditions are present. This is also reflected in the CAP hierarchical triggering structure. The Mood Disturbance CAP was not commonly triggered alone, but when almost all CAPs were triggered, all persons triggered the Mood Disturbance CAP. This suggests that triggering the Mood Disturbance CAP may be related to increased symptom burden. It may also be possible that symptom characteristics such as length of time since onset, intensity, and frequency in relation to disease diagnosis may also affect the degree the symptoms impact on the person's health and QOL.

Palliative care should respond to the needs of persons of all ages. Findings from this study suggest that older persons are not only more complex and likely to exhibit the greatest needs but that with the exception of the Pain CAP and Mood Disturbance CAP, they trigger CAPs more frequently. Age-related barriers to palliative care referral, resource allocation, and service utilization 
in Canada have been reported elsewhere [37-40] and may be compounded by other challenges such as disease diagnosis, and geographic location of care [41]. Therefore, greater investigation into how age affects patterns in CAP triggering is warranted.

While the majority of CAPs were more likely to be triggered among older cohorts, two exceptions were the Mood Disturbance CAP and Pain CAP which younger persons were most likely to trigger. A reason for these discrepancies may be that mood disturbances and pain are not less prevalent in younger age groups but rather that challenges exist for clinicians to recognize these symptoms $[42,43]$. Difficulties with mood and in particular symptoms of depression may also be under-recognized in the older adult population due to their atypical presentation [21,44]. Mood disturbances and depression among older adults may be expressed as physical rather than psychological symptoms such as fatigue, weight loss, or gastro-intestinal problems in contrast to direct communications of feelings of sadness or expressions of depressed mood. This presents unique age-associated challenges for clinicians to recognize the signs of depression and mood disturbance in an older adult population.

The Pain CAP is commonly triggered for those aged 18-44. There are many possible explanations for this. First, younger persons experiencing severe pain or challenges in pain management may feel more confident to voice their concerns over pain management and therefore be referred more often than older adults who may be more hesitant to discuss pain symptoms [45]. The ageist myth, that older adults are used to pain and do not need treatment, may result in pain not being noted or addressed. Older adults may be hesitant to express feelings of pain due to the belief that it is a natural part of the aging process [45]. Rao and Cohen note that lack of recognition of pain symptoms and severity, as well as a lack of understanding of the benefits of pain treatment and management is frequently seen in older persons [46]. Cognitive impairment may affect the ability to communicate pain, challenge the clinician's ability to recognize signs that pain is present, and result in the underreporting of pain [47]. However, it may also reflect a failure of clinicians to recognize pain among the older population. The prevalence of persons exhibiting cognitive impairment increases with age and thereby may also result in an elevated risk for under-recognition and under-treatment of pain for older adults.

Conversely, limitations of the present study should also be recognized. First, the sample is based on volunteer organizations representing some, but not all, regions of Ontario, meaning the results may not be generalizable to the full provincial population or to other jurisdictions. Second, given that the data were drawn from pilot studies prior to the mandated implementation in Ontario there were some issues with missing data for certain interRAI PC items that would generally not occur when a formal reporting system (e.g., Canadian Institute for Health Information's Home Care Reporting System) is in place. This meant that some items that may have been helpful for creating CAP triggers were excluded due to missing data. The section most affected by this issue was the item set dealing with spirituality. Finally, date of death was available only for a subset of pilot study participants so it was not possible to examine associations of CAPs with survival time. This may be addressed in future research because the instrument has now been implemented province wide as the standard assessment for this sector and the data are linkable to administrative records that would include date of death.

It is commonly accepted that as persons near the end of life, the number of health issues and challenges increases. CAP triggering rates differ greatly by age and estimated prognosis. The CAPs do not provide a set treatment plan, but help guide the clinician to consider relevant issues, assist in the prioritization of treatment feasibility, and inform best practice guidelines. In consultation with the person and, when appropriate, members of their informal support network, decisions on whether or not issues raised by the CAPs should be addressed must be made on a case-by-case basis. Wishes expressed by the person should be reflected when assessing treatment burden-benefit and determining whether or not to treat. It is important to remember that even in the final stages of life, persons may respond to and benefit from treatments that decrease symptom burden thereby improving QOL at the end of life.

\section{Conclusion}

Data gathered from the interRAI PC may improve the understanding of the complex needs of palliative home care clients in Ontario and other locations. Patterns in CAP triggering suggest increased attention should be given to address the increasingly complex needs of vulnerable populations. Future research should investigate variation by geographic location and reasons for ageassociated disparities in CAP triggering. Integration of evidence gathered from the interRAI PC CAPs into the care planning process may allow for higher quality of care through better tailoring of resources at address personspecific need.

\section{Competing interests \\ The authors declare that they have no competing interests.}

\section{Authors' contributions}

SF and JPH conceived and designed the study. SF assisted in pilot data collection, conducted the statistical analysis and interpretation of the data, and drafted the manuscript. JPH oversaw pilot data collection, assisted with the statistical analysis and reviewed interpretation of the data, and critically revised all drafts of the manuscript. TFS provided feedback on the design of 
the study and was the lead for all aspects of pilot data collection. KS provided feedback on the design of the study and led creation of the interRAI PC assessment instrument. PS and JG reviewed the study design and critically revised drafts of the manuscript. JPM reviewed interpretation of the data and critically revised drafts of the manuscript. All authors made valuable contributions to the development and refinement of this manuscript and its intellectual content. All authors read and provided approval of the completed manuscript.

\section{Acknowledgements}

The authors wish to acknowledge the clinicians and staff of the Community Care Access Centres in Ontario, Canada for their time and dedication to piloting this instrument and ensuring high quality data collection. The authors thank Dr. Robyn Cohen (McGill University) and Dr. Lora Giangregorio (University of Waterloo) for their constructive feedback on this project. SF wishes to acknowledge the Canadian Institute for Health Research for supporting this research through a Frederick Banting and Charles Best Doctoral Student Award 2009-2013. JPH holds the Ontario Home Care Research and Knowledge Exchange Chair funded by the Ontario Ministry of Health and Long Term Care.

\section{Author details}

${ }^{1}$ School of Health Sciences, University of Northern British Columbia, 3333 University Way, Prince George, British Columbia V2N 4Z9, Canada. ${ }^{2}$ School of Public Health and Health Systems, University of Waterloo, 200 University Ave. West, Waterloo, ON N2L 6P4, Canada. ${ }^{3}$ Department of Sociology, Nipissing University, North Bay, Ontario, Canada. ${ }^{4}$ Retired Chief Emeritus of Geriatrics, Hackensack University Medical Center, 20 Prospect Ave, Hackensack, NJ 07601, USA. ${ }^{5}$ Hebrew Senior Life, 1200 Centre Street, Boston, MA 02131, USA.

Received: 28 August 2014 Accepted: 11 December 2014 Published: 15 December 2014

\section{References}

1. Canadian Hospice Palliative Care Association: A model to guide hospice palliative care. Ottawa, ON. 2013. http://www.chpca.net/professionals/ norms.aspx.

2. World Health Organization: WHO definition of palliative care. 2014 http://www.who.int/cancer/palliative/definition/en/print.html.

3. Pereira J, Bruera E: Alberta hospice palliative care resource manual. Alberta DoPCM-Uo 2001.

4. Salam-White L, Hirdes JP, Poss JW, Blums J: Predictors of emergency room visits or acute hospital admissions prior to death among hospice palliative care clients in Ontario: a retrospective cohort study. BMC Palliat Care 2014, 13(1):35.

5. Melin-Johansson C, Axelsson B, Gaston-Johansson F, Danielson E: Significant improvement in quality of life of patients with incurable cancer after designation to a palliative homecare team. Eur J Cancer Care 2010, 19(2):243-250

6. Steel K, Ljunggren G, Topinková E, Morris JN, Vitale C, Parzuchowski J, Nonemaker S, Frijters DH, Rabinowitz T, Murphy KM, Ribbe MW, Fries BE: The RAI-PC: an assessment instrument for palliative care in all settings. Am J Hosp Palliat Care 2003, 20(3):211-219.

7. Steel K, Smith TF, Morris JN, Freeman S, Hirdes JP, Curtin-Telegdi N, Berg K, Björkgren M, Declercq A, Finne-Soveri H, Fries BE, Frijters D, Gray L, Henrard J-C, Ljunggren G, Szczerbińska K, Topinková E: interRAl Palliative Care Palliative Care Clinical Assessment Protocols (CAPs): For Use with the Palliative Care Assessment Instrument. Version 9.1. Washington, DC: interRAl; 2013.

8. Hirdes JP, Fries BE, Morris JN, Steel K, Mor V, Frijters D, LaBine S, Schalm C, Stones MJ, Teare G, Smith T, Marhaba M, Pérez E, Jónsson P: Integrated health information systems based on the RAI/MDS series of instruments. Healthc Manag Forum 1999, 12:30-40.

9. Hirdes JP, Ljunggren G, Morris JN, Frijters DH, Soveri HF, Gray L, Björkgren $M$, Gilgen R: Reliability of the interRAI suite of assessment instruments: a 12 -country study of an integrated health information system. BMC Health Serv Res 2008, 8(1):277

10. Gray LC, Berg K, Fries BE, Henrard JC, Hirdes JP, Steel K, Morris JN: Sharing clinical information across care settings: the birth of an integrated assessment system. BMC Health Serv Res 2009, 9(1):71.
11. Hirdes JP: Addressing the health needs of frail elderly people: Ontario's experience with an integrated health information system. Age Ageing 2006, 35:329-331.

12. Hirdes JP, Mitchell L, Maxwell CJ, White N: Beyond the 'iron lungs of gerontology': using evidence to shape the future of nursing homes in Canada. Can J Aging 2011, 30(3):371-390.

13. Ontario Association of Community Care Access Centers: OACCAC News: Implementation of electronic palliative assessment tool supported by education. 2012, 11:5. http://oaccac.com/News/Documents/OACCAC News Feb 2012.pdf.

14. Carpenter Gl: Accuracy, validity and reliability in assessment and in evaluation of services for older people: The role of the interRAI MDS assessment system. Age Ageing 2006, 35(4):327-329.

15. Brandeis GH, Berlowitz DR, Hossain M, Morris JN: Pressure ulcers: The minimum data set and the resident assessment protocol. Adv Wound Care 1995, 8(6):18-25.

16. Martin L, Hirdes JP, Morris JN, Montague P, Rabinowitz T, Fries BE: Validating the Mental Health Assessment Protocols (MHAPs) in the Resident Assessment Instrument Mental health (RAI-MH). J Psychiatr Ment Health Nurs 2009, 16(7):646-653.

17. Zhu M, Zhang Z, Hirdes JP, Stolee P: Using machine learning algorithms to guide rehabilitation planning for home care clients. BMC Med Inform Decis Mak 2007, 7(1):41.

18. Resnick NM, Brandeis GH, Baumann MM, Morris JN: Evaluating a national assessment strategy for urinary incontinence in nursing home residents: reliability of the minimum data set and validity of the resident assessment protocol. Neurourol Urodyn 1996, 15(6):583-598.

19. Mathias K, Hirdes JP, Pittman D: A care planning strategy for traumatic life events in community mental health and inpatient psychiatry based on the InterRAI assessment instruments. Community Ment Health J 2010, 46(6):621-627

20. Neufeld E, Perlman CM, Hirdes JP: Predicting inpatient aggression using the interRAl risk of harm to others clinical assessment protocol. J Behav Health Serv Res 2012, 39(4):472-480.

21. Neufeld E, Freeman S, Joling K, Hirdes JP: "When the golden years are blue": Changes in depressive symptoms over time among older adults newly admitted to long-term care facilities. Clin Gerontol 2014 37(3):1-18.

22. Steel K, Morris JN, Leff B: Dyspnea Cap. In interRAl Palliative Care Palliative Care Clinical Assessment Protocols (CAPS): For Use with the Palliative Care Assessment Instrument. Version 9.1. Edited by Steel K, Smith TF, Morris JN, Freeman S, Hirdes JP, Curtin-Telegdi N, Berg K, Björkgren M, Declercq A, Finne-Soveri H, Fries BE, Frijters D, Gray L, Henrard J-C, Ljunggren G, Szczerbińska K, Topinková E. Washington, DC: interRAl; 2013:7-12.

23. Steel K, Inouye SK, Morris JN, Murphy KM, Marcantonio E: Delirium Cap. In interRAl Palliative Care Palliative Care Clinical Assessment Protocols (CAPs): For Use with the Palliative Care Assessment Instrument. Version 9.1. Edited by Steel K, Smith TF, Morris JN, Freeman S, Hirdes JP, Curtin-Telegdi N, Berg K, Björkgren M, Declercq A, Finne-Soveri H, Fries BE, Frijters D, Gray L, Henrard J-C, Ljunggren G, Szczerbińska K, Topinková E. Washington, DC: interRAl; 2013:12-15.

24. Olsen K, Steel K, Ljunggren G, Steel E, Smith TF: Fatigue Cap. In interRAl Palliative Care Palliative Care Clinical Assessment Protocols (CAPs): For Use with the Palliative Care Assessment Instrument. Version 9.1. Edited by Steel K, Smith TF, Morris JN, Freeman S, Hirdes JP, Curtin-Telegdi N, Berg K, Björkgren M, Declercq A, Finne-Soveri H, Fries BE, Frijters D, Gray L, Henrard J-C, Ljunggren G, Szczerbińska K, Topinková E. Washington, DC: interRAl; 2013:13-20.

25. Won A, Finne-Soveri H, Frijters D, Gambassi G, Murphy KM, Morris JN, Steel K, Steel E, Gravell D: Pain Cap. In interRAl Palliative Care Palliative Care Clinical Assessment Protocols (CAPs): For Use with the Palliative Care Assessment Instrument. Version 9.1. Edited by Steel K, Smith TF, Morris JN, Freeman S, Hirdes JP, Curtin-Telegdi N, Berg K, Björkgren M, Declercq A, Finne-Soveri H, Fries BE, Frijters D, Gray L, Henrard J-C, Ljunggren G, Szczerbińska K, Topinková E. Washington, DC: interRAl; 2013:35-42.

26. Smith TF, Rabinowitz T, Hirdes JP, Morris JN, Stewart S, Constantino C, Stee K: Mood Cap. In interRAl Palliative Care Palliative Care Clinical Assessment Protocols (CAPS): For Use with the Palliative Care Assessment Instrument Version 9.1. Edited by Steel K, Smith TF, Morris JN, Freeman S, Hirdes JP, Curtin-Telegdi N, Berg K, Björkgren M, Declercq A, Finne-Soveri H, Fries BE, Frijters D, Gray L, Henrard J-C, Ljunggren G, Szczerbińska K, Topinková E. Washington, DC: interRAl; 2013:26-31. 
27. Ljunggren G, Olson K, Smith TF, Steel K, Hirdes JP, Morris JN: Sleep Disturbance Cap. In interRAl Palliative Care Palliative Care Clinical Assessment Protocols (CAPs): For Use with the Palliative Care Assessment Instrument. Version 9.1. Edited by Steel K, Smith TF, Morris JN, Freeman S, Hirdes JP, Curtin-Telegdi N, Berg K, Björkgren M, Declercq A, Finne-Soveri H, Fries BE, Frijters D, Gray L, Henrard J-C, Ljunggren G, Szczerbińska K, Topinková EL. Washington: interRAl; 2013:51-56.

28. Steel K, Morris JN, Sorby LW, Steel E: Nutrition Cap. In interRAI Palliative Care Palliative Care Clinical Assessment Protocols (CAPS): For Use with the Palliative Care Assessment Instrument. Version 9.1. Edited by Steel K, Smith TF, Morris JN, Freeman S, Hirdes JP, Curtin-Telegdi N, Berg K, Björkgren M, Declercq A, Finne-Soveri H, Fries BE, Frijters D, Gray L, Henrard J-C, Ljunggren G, Szczerbińska K, Topinková E. Washington, DC: interRAl; 2013:28-364.

29. Steel $K$, Leff $B$, Brandeis $G H$, Finne-Soveri $H$, Morris JN, Nonemaker $S$, Szczerbińska K, Beleville-Taylor P: Pressure Ulcer Cap. In interRAl Palliative Care Palliative Care Clinical Assessment Protocols (CAPs): For Use with the Palliative Care Assessment Instrument. Version 9.1. Edited by Steel K, Smith TF, Morris JN, Freeman S, Hirdes JP, Curtin-Telegdi N, Berg K, Björkgren M, Declercq A, Finne-Soveri H, Fries BE, Frijters D, Gray L, Henrard J-C, Ljunggren G, Szczerbińska K, Topinková E. Washington, DC: interRAl; 2013:43-50.

30. White C, McMullan D, Doyle J: "Now that you mention it, doctor...": Symptom reporting and the need for systematic questioning in a specialist palliative care unit. J Palliat Med 2009, 12(5):447-450.

31. Lorenz KA, Lynn J, Dy SM, Shugarman LR, Wilkinson A, Mularski RA, Morton SC, Hughes RG, Hilton LK, Maglione M, Rhodes SL, Rolon C, Sun VC, Shekelle PG: Evidence for improving palliative care at the end of life: A systematic review. Ann Intern Med 2008, 148(2):147-159.

32. Seow H, King S, Vaitonis V: The impact of Ontario's end-of-life care strategy on end-of-life care in the community. Health Care Q 2008, 11(1):56-61.

33. McClement S: Acquiring an evidence base in palliative care: Challenges and future directions. Expert Rev Pharmacoecon Outcomes Res 2006, 6(1):37-40.

34. Shugarman LR, Lorenz K, Lynn J: End-of-life care: An agenda for policy improvement. Clin Geriatr Med 2005, 21:255-272.

35. Lorenz KA, Shugarman LR, Lynn J: Health care policy issues in end-of-lifecare. J Palliat Med 2006, 9(3):731-748.

36. Edmonds P, Karlsen S, Khan S, Addington-Hall J: A comparison of the palliative care needs of patients dying from chronic respiratory diseases and lung cancer. Palliat Med 2001, 15(4):287-295.

37. Burt J, Raine R: The effect of age on referral to and use of specialist palliative care services in adult cancer patients: A systematic review. Age Ageing 2006, 35(5):469-476.

38. Burge Fl, Lawson B, Critchley P, Maxwell D: Transitions in care during the end of life: changes experienced following enrolment in a comprehensive palliative care program. BMC Palliat Care 2005, 4(1):3

39. Burge Fl, Lawson BJ, Johnston GM, Grunfeld E: A population-based study of age inequalities in access to palliative care among cancer patients. Med Care 2008, 46(12):1203-1211.

40. NELS ICE: End of Life Care in Nova Scotia Surveillance Report. Halifax: Network for End of Life Studies (NELS) Interdisciplinary Capacity Enhancement (ICE); 2008. http:/www.dal.ca/content/dam/dalhousie/pdf/sites/nels/ report_ICESurveillance2008.pdf.

41. Freeman S, Heckman G, Naus PJ, Marston HR: Breaking down barriers: Hospice palliative care as a human right in Canada. Educ Gerontol 2013, 39(4):241-249.

42. Proctor WR, Hirdes JP: Pain and cognitive status among nursing home residents in Canada. Pain Res Manag 2000, 6(3):119-125.

43. Życzkowska J, Szczerbińska K, Jantzi MR, Hirdes JP: Pain among the oldest old in community and institutional settings. Pain 2007, 129(1):167-176.

44. Szczerbińska K, Hirdes JP, Życzkowska J: Good news and bad news: depressive symptoms decline and undertreatment increases with age in home care and institutional settings. Am J Geriatr Psychiatry 2012, 20(12):1045-1056
45. Bernabei R, Gambassi G, Lapane K, Landi F, Gatsonis C, Dunlop R, Lipsitz L, Steel K, Mor V: Management of pain in elderly patients with cancer. JAMA 1998, 279(23):1877-1882.

46. Rao A, Cohen HJ: Symptom management in the elderly cancer patient: fatigue, pain, and depression. J Natl Cancer Inst 2004, 32:150-157.

47. Brazil K: Palliative care for the older adult. In A public health perspective on end of life care. Edited by Cohen J, Deliens L. Oxford: University Press; 2012.

\section{doi:10.1186/1472-684X-13-58}

Cite this article as: Freeman et al.: Care planning needs of palliative home care clients: Development of the interRAI palliative care assessment clinical assessment protocols (CAPs). BMC Palliative Care 2014 13:58.

\section{Submit your next manuscript to BioMed Central and take full advantage of:}

- Convenient online submission

- Thorough peer review

- No space constraints or color figure charges

- Immediate publication on acceptance

- Inclusion in PubMed, CAS, Scopus and Google Scholar

- Research which is freely available for redistribution 